\title{
Properties of metallic nanocrystalline powders
}

Ivo Marek, Alena Michalcova, Dalibor Vojtech

Department of Metals and Corrosion Engineering, Institute of Chemical Technology, Prague, Technicka 5, 166 28, Prague 6, Czech Republic. Ivo.Marek@vscht.cz

Nanocrystalline metals are advanced materials with structural constituents smaller than $100 \mathrm{~nm}$. With respect to structure, they are characterized by high strength and hardness, gas absorption capability, high specific surface area, catalytic activity or magnetic properties. These properties predispose them for using in wide spectrum of possible applications, namely advanced structural and functional applications, biomedicine, catalysis or electronics. Many methods have been developed for producing nanocrystalline metals or nanocrystalline metallic powders including precipitation from supersaturated liquids, severe plastic deformation, cryo-melting, or inert gas condensation. In this work, another promising method - selective dissolving of aluminium alloy was successfully appliedfor preparation of nanocrystalline copper powder and this powder was consequently used for producing of bulk nanocrystalline material with enhanced hardness and strength.

Keywords: nanocrystalline metals, metallic powders, structure, hardness

\section{Acknowledgements}

Research on nano-crystalline metals is financially supported by the Czech Science Foundation (project no. P108/12/G043).

\section{References}

[1] LU, K. (1996). Nanocrystalline metals crystallized from amorphous solids: nanocrystallization, structure, and properties.Materials Science and Engineering $R$, Vol. 16, pp. 161-221.

[2] LIN, H.-M., HSIEH, S.-H., LEE, P.Y., LAI, M.-S., WU, J.S. (1993). Synthesis and characterization of nanocrystalline powders of pure nickel and copper.Material Chemistry and Physics, Vol. 34, pp. 205-213.

[3] BIRRINGER, R. (1989). Nanocrystalline materials.Materials Science and EngineeringA, Vol. 117, pp. 33-43.

[4] TJONG, S.C., HAYDN, Ch. (2004). Nanocrystalline materials and coatings. Materials Science and Engineering $R$, Vol. 45, pp. 1-88.

[5] MEYERS, M.A., MISHRA, A., BENSON, D.J. (2006). Mechanical properties of nanocrystalline materials. Progress in Materials Science, Vol. 51, pp. 427-556.

[6] SANDERS, P.G., EASTMAN, J.A., WEERTMAN, J.R. (1997). Elastic and tensile behavior of nanocrystalline copper and palladium. Acta Materialia, Vol. 45, No. 10, pp. 4019-4025.

[7] CHEN, S.-J., LI, X.-B., NIU, G., YI, Z., CHEN, Y., LUO, J.-S., TANG, Y.-J., SUN, W.-G. (2012). Synthesis and characterization of single-phase nanocrystalline $\mathrm{Ag}_{2} \mathrm{Al}$ particles. Transactions of Nonferrous Metals Society of China, Vol. 22, pp. 134-138.

[8] KUMAR, K.S., VAN SWYGENHOVEN, H., SURESH, S. (2003). Mechanical behavior of nanocrystalline metals and alloys. Acta Materialia, Vol. 51, pp. 5743-5774.

[9] HANLON, T., KWON, Y.-N., SURESH, S. (2003). Grain size effects on the fatigue response of nanocrystalline metals. Scripta Materialia, Vol. 49, pp.675-680.

[10] SURYANARAYANA, C., KOCH, C.C., (2000). Nanocrystalline materials - Current research and future directions. Hyperfine Interactions, Vol. 130, pp. 5-44.

[11] GLEITER, H. (1989). Nanocrystalline materials. Progress in Materials Science, Vol. 33, pp. 223-315.

[12] GUTMANAS, E.Y., TRUSOV, L.I., GOTMAN, I. (1994). Consolidation, microstructure and mechanical properties of nanocrystalline metal powders. NanoStructured Materials, Vol. 4, No. 8, pp. 893-901.

[13] HUANG, Y.K., MENOVSKY, A.A., de BOER, F.R. (1993). Electrical resistivity of nanocrystalline copper. NanoStructured Materials, Vol. 2, No. 5, pp. 505-513.

[14] DREIZIN, E.L. (2009). Metal-based reactive nanomaterials. Progress in Energy and Combustion Science, Vol. 35, pp. 141-167.

[15] CHAMPION, Y., BIGOT, J. (1998). Synthesis and structural analysis of aluminium nanocrystalline powders. 
NanoStructured Materials, Vol. 10, No. 7, pp. 1097-1110.

[16] SURYANARAYANA, C. (1995). Nanocrystalline materials. International Materials Reviews, Vol. 40, No. 2 , pp. 41-64.

[17] MICHALCOVA, A., VOJTECH, D. (2012). Structure of rapidly solidified aluminium alloys. Manufacturing Technology, Vol. 12, pp. 166-169.

[18] NOVA, I., MACHUTA, J. (2012). Squeeze casting results of aluminium alloys. Manufacturing Technology, Vol. 12, pp. 73-79.

[19] VOJTECH, D., MICHALCOVA, A., KNOTEK, V., MAREK, I. (2012). Study of nano-crystalline metals prepared by selective chemical leaching. Manufacturing Technology, Vol. 12, pp. 292-296.

[20] MEYERS, M.A., CHAWLA, K.K. (2009). Mechanical behavior of materials. CAMBRIDGE UNIVERSITY PRESS. 